\title{
La ciencia política en Venezuela: FORTALEZAS PASADAS Y VULNERABILID ADES PRESENTES
}

\author{
Ángel Álvarez Díaz \\ Universidad Central de Venezuela, Venezuela \\ Said Dahdah Antar \\ Universidad Central de Venezuela, Venezuela
}

\begin{abstract}
Resumen
Este artículo presenta un panorama de la ciencia política en Venezuela. Se describen la evolución de la disciplina en el país y se hace una evaluación de sus fortalezas y debilidades. El perfil de la disciplina toma en cuenta la evolución teórica y un mapa metodológico que considera las principales líneas de división. También se muestra el desarrollo institucional de la investigación y la enseñanza de la politología en Venezuela, así como las principales tendencias en el mercado de trabajo. Se concluye que la institucionalización de la disciplina está actualmente amenazada por las crisis económica y política que vive el país.
\end{abstract}

\begin{abstract}
This paper presents an overview of the main trends in Venezuelan political science. It describes the domestic evolution of the discipline and it also assesses the strengths and weaknesses of the Venezuelan political science scholarship. This article addresses both the theoretical evolution and the main methodological debates. It also shows the institutional development of both research and teaching, along with the main trends in the job market. We conclude that the process of institutionalization of the discipline is currently threatened by both economic and political crisis.
\end{abstract}

PALABRAS CLAVE • Ciencia Política • Venezuela • Teoría • Institucionalización • Publicaciones • Politólogos

\section{INTRODUCCIÓN}

La ciencia política es la disciplina académica de más reciente creación en las universidades venezolanas. El primer centro de investigaciones, el Instituto de Estudios Políticos de la Universidad Central de Venezuela, UCV, fue fundado en 1958. La primera escuela de ciencia política se inaugura en Caracas, en la misma universidad, hace poco más de 30 años. En las últimas tres décadas, el estudio de la disciplina se ha extendido y multiplicado por todo el país, tanto al nivel de licenciatura como al nivel de postgrado. Igualmente han crecido tanto la cantidad de estudiantes como la cifra de publicaciones especializadas, así como se han diversificado corrientes de investigación.

Crecimiento, no obstante, no significa maduración. Factores institucionales, financieros, de enfoque y, más recientemente, de entorno político y financiero han afectado la cantidad y la calidad de 
la investigación y la docencia. Luego de un notable crecimiento especialmente pronunciado en las décadas de los 80 y 90, hoy en día parecen haber razones de fondo para pensar que la ciencia política en Venezuela se encuentra en una encrucijada. Su independencia y, en consecuencia, su crecimiento como disciplina auténticamente científica, están amenazados por las crisis políticas y económicas que afectan a la sociedad en su conjunto. Por ello, su futura consolidación como disciplina científica autónoma no va a depender sólo de los actores académicos que hoy en día ejercen la profesión y lideran el oficio; dependerá también, y en mucho, de factores exógenos que el mundo universitario no puede controlar, ni predecir con certeza.

En las próximas líneas se presenta un apretado sumario de la historia, los logros, fallas y los retos de la ciencia política venezolana. Se muestran las diferencias y puntos de coincidencia de las diferentes escuelas y centros de investigación existentes en el país, así como las posibilidades de integración. Concluyendo con un balance de los logros y faltas, así como con una llamada de atención sobre el futuro inmediato de la disciplina en un contexto que pudiera llegar a serle adverso.

\section{ORIGEN HISTÓRICO Y EVOLUCIÓN TEÓRICA DE LA CIENCIA POLÍTICA VENEZOLANA}

La fecha y lugar de nacimiento de nuestra disciplina no podía ser más propicia. La ciencia política nace con la democracia venezolana, en 1958, y en la universidad más politizada y comprometida con las luchas por la democracia: la Universidad Central de Venezuela, UCV. Desde ese momento, el aumento, el desarrollo y el fortalecimiento de la disciplina han dependido del nivel de desarrollo democrático del país. Es cierto que la evolución de toda ciencia se favorece con el clima de libertades que auspicia la democracia, pero la ciencia política es tal vez la especialidad académica que más depende del clima de libertad de expresión y de tolerancia a la crítica. Así entonces, la ciencia política venezolana nace y crece al abrigo del desarrollo progresivo de libertades cívicas y de la institucionalización de la autonomía universitaria.

El primer centro de investigación, el Instituto de Estudios Políticos (IEP), es fundado en la Facultad de Ciencias Jurídicas y Políticas de la UCV. Su creación y dirección es encargada al destacado constitucionalista español Manuel García Pelayo. Antes de la creación del IEP, la enseñanza embrionaria de la disciplina se redujo al estudio del derecho constitucional en las escuelas de derecho de las universidades autónomas. Desde su creación hasta el presente, el IEP aborda y profundiza el estudio del derecho constitucional venezolano y comparado, pero también de la historia del pensamiento y de las formas políticas, de la filosofía política y de las relaciones internacionales.

La tarea docente del IEP se concentró, por décadas, en seminarios dirigidos a estudiantes de derecho a nivel de licenciatura y en cursos de postgrado en el doctorado en derecho público. Estos cursos irán poniendo los cimientos para la creación de un cuerpo de investigadores y docentes que, en el transcurso de poco más de una década, serán el soporte de las primeras publicaciones especializadas y las primeras de pregrado y postgrado. En 1971, el IEP crea la primera revista académica de ciencia política: el anuario POLITEIA. Luego, en 1973, se crea la Escuela de Estudios Políticos y Administrativos (EEPA), para la enseñanza que otorga el título de Licenciado en Estudios Políticos en tres sub-especialidades: politología, relaciones internacionales y administración pública. A nivel de postgrado, el Doctorado en Derecho Público es transformado en Doctorado en Ciencias 
Políticas. Así, esos tres entes académicos (el IEP, la EEPA y el Doctorado en Derecho) junto a POLITEIA serán los pilares del inicio del proceso de institucionalización de la disciplina.

La ciencia política venezolana, en sus orígenes, tuvo dos rasgos fundamentales: primero, un deliberado empeño por evitar el "parroquianismo" y, segundo, un fuerte acento histórico-institucional. La primera característica condujo a una tal vez exagerada concentración en el estudio de la realidad institucional europea y al descuido de la realidad venezolana y latinoamericana. El segundo rasgo, llevó a la creación de una fuerte corriente historicista en desmedro de los métodos de investigación empírica.

A finales de los 70 se produce una fuerte reacción anti-historicista y más favorable al estudio de la realidad Latinoamericana y la venezolana, empleando de forma incipiente el razonamiento de teoría de juegos. En el seno del propio IEP, J uan Carlos Rey lidera el desarrollo de una línea de investigación que va a crear escuela: el estudio del sistema político venezolano (SPV) y, particularmente, del sistema de partidos, desde una perspectiva empírica. La visita a Venezuela, por los años 70, de cientistas políticos norteamericanos, nombradamente Daniel Levine y Enrique Baloyra ${ }^{1}$, también influirá en este giro de la ciencia política venezolana hacia problemas más inmediatos. Como consecuencia, el enfoque conductista, en primer lugar y por poco tiempo, y más tarde y con más impacto el enfoque neo-institucional, van a influir profundamente en la formación de los estudiantes de las primeras promociones de la Escuela de Estudios Políticos.

De igual modo, desde mediados de los 70 , la ciencia política venezolana va a sufrir un importante y positivo "shock externo". Como consecuencia del quiebre de las democracias del cono sur del continente, un número importante de destacados profesores argentinos, uruguayos y chilenos migran a Venezuela y traen consigo el énfasis en los estudios de América Latina desde una perspectiva comparada. Paralelamente, en la misma UCV, el Centro de Estudios del Desarrollo (CENDES), venía produciendo importantes estudios investigaciones sobre valores políticos de los venezolanos, bajo la dirección de J osé Agustín Silva Michelena².

La convergencia de estos tres factores (un nuevo interés por la política venezolana en el IEP, la influencia de profesores extranjeros, tanto latinoamericanos como norteamericanos, y los trabajos del CENDES) van a revolucionar la enseñanza y la investigación de la ciencia política en el país. El cambio fue profundo y duradero: desde entonces, el énfasis en los problemas venezolanos y, en menor medida de los latinoamericanos, va a marcar la producción bibliográfica de los politólogos domésticos.

Esta tendencia produce, a la larga, dos efectos que aún están presentes. De un lado, una mayor atención a los problemas domésticos de estabilidad, cambio y desarrollo político. En segundo lugar, una incipiente preocupación por el uso de modelos formales y métodos empíricos. No obstante, a la larga, la preocupación por el estudio del SPV ha conducido a un excesivo "parroquialismo". Por su parte, el virtual abandono de los métodos de investigación histórica no ha sido compensado con un fuerte desarrollo de la investigación empírica en la UCV.

Esta tendencia a enfatizar, los problemas domésticos no ha desaparecido pese a la creación de otros muy importantes centros de investigación y docencia tanto en Caracas (la Maestría en Ciencias Políticas de la Universidad Simón Bolívar, USB, y el Área de Políticas Públicas del Instituto 
de Estudios Superiores de la Administración, IESA), así como en otras ciudades del país (la Escuela de Estudios Políticos de la Universidad de los Andes, ULA, y el Doctorado y el Instituto de Ciencias Políticas de la Universidad del Zulia, LUZ). Por el contrario, pese a la multiplicidad de centros de enseñanza y de investigación (ver Tabla 4 y 5), durante los últimos 25 años la ciencia política se ha estado haciendo básicamente una misma pregunta: ¿Qué explica la estabilidad 0 , desde 1993, la inestabilidad de la democracia venezolana?

Las respuestas, sin embargo, han variado de escuela a escuela. Mientras en el IEP de la UCV la respuesta predominante ha sido de carácter institucional, usando básicamente herramientas analíticas neo-institucionales e histórico-institucionales, la respuesta predominante en el CENDES ha sido de tipo estructural y sociológico. Mientras el IEP ha enfatizado el rol de las reglas de juego para manejar el disenso y construir lo que Rey bautizó como el "modelo populista de conciliación"3 y las razones históricas de la consolidación y crisis del sistema político ${ }^{4}$, el CENDES ha privilegiado siempre la asimetría de poder y el conflicto entre grupos sociales económica y culturalmente heterogéneos ${ }^{5}$. En una línea parcialmente coincidente, Steve Ellner, desde la Universidad de Oriente (UDO), ha aportado explicaciones estructurales tanto de la estabilidad pasada como de la crisis del sistema político venezolano ${ }^{6}$. Por otro lado, en la USB, los trabajos de Arístides Torres y, hoy en día, los de Federico Welsh y J osé Vicente Carrasqueño, han enfatizado la búsqueda de respuestas en las actitudes y la cultura política ${ }^{7}$. En el IESA, por su propia naturaleza como centro de estudios de políticas públicas, el énfasis ha estado siempre en las capacidades de gestión del Estado, dados ciertos constreñimientos económicos (básicamente el modelo petrolero-rentista y proteccionista de crecimiento ${ }^{8}$ ) e institucionales (el excesivo centralismo). Finalmente, en la LUZ, José Enrique Molina ha mostrado la importancia de las reglas electorales, así como la institucionalización y la desinstitucionalización del sistema de partidos ${ }^{9}$.

\section{DESARROLLO INSTITUCIONAL DE LA INVESTIGACIÓN Y LA ENSEÑANZA DE LA CIEN- CIA POLÍTICA EN VENEZUELA}

\section{Las Publicaciones Científicas}

En la actualidad, la investigación y enseñanza de ciencia política en Venezuela cuenta con una oferta de espacios de publicación. Once revistas hacen posible la exposición y el debate de las propuestas de los cientistas políticos. Los 374 ejemplares registrados en la Tabla 1, con una periodicidad que se concentra entre lo semestral y cuatrimestral, abandonando la anualidad de los comienzos, indican la presencia de una producción editorial que permite la expresión y, en consecuencia, el conocimiento de los enfoques manejados por las distintas orientaciones para explicar la realidad política. En este

Rey, J uan Carlos (1972).

Urbaneja, Diego Bautista (1978).

Silva Michelena, J osé A. y Heinz R. Sonntag, (1979); J osé A. (coord.) (1987); Sonntag, Heinz R. y Thaís, Maingón (1992);

López Maya, Margarita (1989).

Ellner, Steve y D. Hellinger (eds.) (2003); Ellner, Steve (1998).

Arístides (1982); Welsh, Federico (1992); Jose Vicente (1994).

Naim, Moisés y Ramón Piñango (1984); Kelly, J aneth (2003); Kelly, J aneth (1995).

Molina, José E. y Carmen Pérez Baralt (1999); Molina, J osé E. y Carmen Pérez Baralt (1998). 
sentido, es necesario precisar que, aunque el promedio de tiraje sea de mil ejemplares por edición, el sistema de distribución de los mismos presenta deficiencias considerables.

Politeia y la Revista Venezolana de Ciencias Políticas, por ejemplo, son revistas donde predominan los trabajos neo-institucionales e histórico-institucionales. Cuadernos del Cendes, la Revista Venezolana de Ciencias Políticas, la Revista de Ciencias Sociales y la Revista Venezolana de Ciencias Sociales son espacios editoriales propios del enfoque estructural y de evaluación de las políticas públicas. Cuestiones Políticas es una publicación con una tendencia favorable a los estudios empíricos y a la evaluación de las condiciones del sistema político, de partido y electoral. Una revista como Ciencias del Gobierno, finalmente, presenta trabajos que comprenden el área de las políticas públicas.

La magnitud de la producción de revistas en Venezuela obedece, en buena medida, a la presencia de una política pública patrocinada por el Fondo Nacional de Ciencia, Tecnología e Innovación (FONACIT), institución adscrita al Ministerio de Ciencia y Tecnología. El Registro de Publicaciones Científicas es el programa de financiamiento anual de esta clase de revistas. De hecho, las diez publicaciones presentadas en la Tabla 1 están acreditadas en el Registro del Núcleo Básico de Publicaciones Científicas y Tecnológicas del FONACIT ${ }^{10}$. Los Consejos de Desarrollo Científico y Humanístico de las Universidades y las Facultades también contribuyen al financiamiento de las publicaciones científicas. En cualquiera de los casos, éstas se mantienen o propician el apego a los lineamientos que caracterizan la política del FONACIT en la materia. 
TABLA 1: Publicaciones indexadas

\begin{tabular}{|c|c|c|c|c|c|c|}
\hline Nombre & $\begin{array}{l}\text { Institución de } \\
\text { adscripción }\end{array}$ & Editor & Periodicidad & $\begin{array}{l}\text { Volú- } \\
\text { menes }\end{array}$ & $\begin{array}{l}\text { Nú- } \\
\text { meros }\end{array}$ & Acesible online web \\
\hline $\begin{array}{l}\text { iencias de } \\
\text { jobierno }\end{array}$ & $\begin{array}{l}\text { Instituto Zuliano de } \\
\text { Estudios Políticos, } \\
\text { Económicos y Sociales } \\
\text { de la Gobernación del } \\
\text { Estado Zulia }\end{array}$ & $\begin{array}{l}\text { Guillermo } \\
\text { García }\end{array}$ & Semestral & 7 & 14 & $\begin{array}{l}\text { http:// www. izepes. } \\
\text { org.ve }\end{array}$ \\
\hline $\begin{array}{l}\text { Cuadernos del } \\
\text { Cendes }\end{array}$ & $\begin{array}{l}\text { Centro de Estudios del } \\
\text { Desarrollo de la } \\
\text { Universidad Central de } \\
\text { Venezuela }\end{array}$ & I Nelly Arenas & Cuatrimestral & 21 & 57 & $\begin{array}{l}\text { http:// www.cendes- } \\
\text { ucv.edu.ve }\end{array}$ \\
\hline $\begin{array}{l}\text { Cuestiones } \\
\text { Políticas }\end{array}$ & $\begin{array}{l}\text { Instituto de Estudios } \\
\text { Políticos y Derecho } \\
\text { Público de la } \\
\text { Universidad del Zulia }\end{array}$ & $\begin{array}{l}\text { Edith Mabel } \\
\text { Cuñarro Conde }\end{array}$ & Semestral & & 33 & $\begin{array}{l}\text { http:// www.serbi.luz. } \\
\text { edu.ve/ revistas/cp/ } \\
\text { eaboutj.htm }\end{array}$ \\
\hline $\begin{array}{l}\text { Espacio Abierto } \\
\text { Cuaderno } \\
\text { Venezolano de } \\
\text { Sociología }\end{array}$ & $\begin{array}{l}\text { Asociación Civil } \\
\text { Espacio Abierto } \\
\text { Investigadores } \\
\text { Asociados }\end{array}$ & $\begin{array}{l}\text { María Cristina } \\
\text { Parra-Sandoval } \\
\text { y Alexis Romero } \\
\text { Salazar }\end{array}$ & Cuatrimestral & 13 & 42 & $\begin{array}{l}\text { http:// www.fonacit. } \\
\text { gov.ve/ publicaciones/ } \\
\text { indice.asp }\end{array}$ \\
\hline $\begin{array}{l}\text { Fermentum, } \\
\text { Revista } \\
\text { Venezolana de } \\
\text { Sociología y } \\
\text { Antropología }\end{array}$ & $\begin{array}{l}\text { HUMANIC, Centro de } \\
\text { Investigaciones en } \\
\text { Ciencias Humanas. } \\
\text { Facultad de } \\
\text { Humanidades, de la } \\
\text { Universidad de } \\
\text { Los Andes }\end{array}$ & $\begin{array}{l}\text { Oscar } \\
\text { Aguilera }\end{array}$ & Cuatrimestral & 11 & 31 & $\begin{array}{l}\text { http:// www. saber.ula. } \\
\text { ve/ humanic/ } \\
\text { fermentum.htm }\end{array}$ \\
\hline Politeia & $\begin{array}{l}\text { Institutos de Estudios } \\
\text { Políticos de la } \\
\text { Universidad Central } \\
\text { de Venezuela }\end{array}$ & $\begin{array}{l}\text { Luis } \\
\text { Salamanca y } \\
\text { Geraldine } \\
\text { León }\end{array}$ & Semestral & & 31 & $\begin{array}{l}\text { http:// www.fonacit. } \\
\text { gov.ve/ publicaciones/ } \\
\text { indice.asp }\end{array}$ \\
\hline $\begin{array}{l}\text { Revista de } \\
\text { Ciencias } \\
\text { Sociales }\end{array}$ & $\begin{array}{l}\text { Instituto de } \\
\text { Investigaciones de la } \\
\text { Universidad del Zulia }\end{array}$ & $\begin{array}{l}\text { Caterina } \\
\text { Clemenza } \\
\text { de Araujo }\end{array}$ & Cuatrimestral & 10 & 30 & $\begin{array}{l}\text { http:// www. serbi.luz. } \\
\text { edu.ve/ revistas/rcs/ } \\
\text { eaboutj.htm }\end{array}$ \\
\hline $\begin{array}{l}\text { Revista } \\
\text { Venezolana de } \\
\text { Ciencia Política }\end{array}$ & $\begin{array}{l}\text { Centro de Estudios } \\
\text { Políticos y Sociales de } \\
\text { América Latina de la } \\
\text { Universidad de los Andes }\end{array}$ & $\begin{array}{l}\text { Alfredo Ramos } \\
\text { Jiménez } \\
\text { es }\end{array}$ & Semestral & 6 & 12 & $\begin{array}{l}\text { http:// www.fonacit. } \\
\text { gov.ve/ publicaciones/ } \\
\text { indice.asp }\end{array}$ \\
\hline $\begin{array}{l}\text { Revista } \\
\text { Venezolana de } \\
\text { Ciencias } \\
\text { Sociales }\end{array}$ & $\begin{array}{l}\text { Vicerrectorado } \\
\text { Académico de la } \\
\text { Universidad Nacional } \\
\text { Experimental Rafael } \\
\text { María Baralt }\end{array}$ & $\begin{array}{l}\text { Edgar Córdova } \\
\text { Jaimes }\end{array}$ & Sen & 4 & 8 & $\begin{array}{l}\text { http:// www.fonacit. } \\
\text { gov.ve/ publicaciones/ } \\
\text { indice.asp }\end{array}$ \\
\hline $\begin{array}{l}\text { Revista } \\
\text { Venezolana de } \\
\text { Economía y } \\
\text { Ciencias } \\
\text { Sociales }\end{array}$ & $\begin{array}{l}\text { Facultad de Ciencias } \\
\text { Económicas y } \\
\text { Sociales de la } \\
\text { Universidad Central } \\
\text { de Venezuela }\end{array}$ & $\begin{array}{l}\text { Margarita } \\
\text { López } \\
\text { Maya }\end{array}$ & Cuatrimestral & 11 & 41 & $\begin{array}{l}\text { http:// www.revele. } \\
\text { com.ve/ }\end{array}$ \\
\hline $\begin{array}{l}\text { Tierra Firme. } \\
\text { Revista de } \\
\text { Historia y } \\
\text { Ciencias Sociales }\end{array}$ & Fundación Tierra Firme & $\begin{array}{l}\text { Arístides } \\
\text { Medina Rubio } \\
\text { y otros }\end{array}$ & Trimestral & 19 & 75 & $\begin{array}{l}\text { http:// www.fonacit. } \\
\text { gov.ve/ publicaciones/ } \\
\text { indice.asp }\end{array}$ \\
\hline
\end{tabular}


En cuanto al formato y contenido, las exigencias apuntan hacia el cumplimiento de la periodicidad en la publicación; la presentación de los trabajos con resúmenes y con palabras clave en español e inglés; la exposición de un índice de contenido interno y de otro en la contraportada; la difusión regular de índices acumulativos y la señalización de las fechas de recepción y aceptación de los trabajos; el ajuste de las citas y referencias bibliográficas a pautas establecidas; y una breve reseña del perfil de cada colaborador. Por otro lado, sólo se publican trabajos inéditos y que no hayan sido presentados simultáneamente a otras revistas. Además, tales trabajos se someten al arbitraje, de acuerdo con el sistema doble ciego. El arbitraje, sujeto a unas reglas, puede ser de uno, al menos dos o hasta ser realizado por un comité de árbitros especialistas.

Finalmente, las revistas acreditadas están presentes en índices como: 1) Clase, de la UNAMMéxico; 2) el Dare Data Bank, de la UNESCO; 3) el Censo de Revistas Iberoamericanas de Cultura, de la Asociación de Revistas Culturales de España; 4) el Servicio de Información al día en Gestión Pública del CLAD; 5 ) el Índice y Directorio de Revistas Venezolanas de Ciencia y Tecnología (REVENCYT); 6) el Ulrich's Internacional Periodicals Directory; 7) Internacional Political Science Abstracts; 8) el Sistema de Registro de Información en Línea para Revistas Científicas de América Latina, el Caribe, España y Portugal (Latindex); 9) la Red de Información Socio-Económica (REDINSE); 10) el Índice Español de Ciencia y Tecnología (ICYT); 11) Sociological Abstracts; 12) el Documentation Politique Internationale Paris IPSA-AISP; 13) Hispanic American Periodical Index USA (HAPI); 14) Fondo Bibliográfico de América Latina (FOBAL).

Sobre esto último, es necesario precisar que, como parte de la política que ejecuta el Centro Nacional de Tecnología e Información (CNTI), todas las publicaciones que constituyen el Registro de Publicaciones Científicas del FONACIT, y por tanto las revistas de la Tabla 1, formarán parte del Scientific Electronic Library Online (Scielo). De hecho, Scielo incluye a Venezuela en la actualidad bajo la figura de iniciativas en desarrollo $0^{11}$.

Según el Reglamento del Programa de Promoción del Investigador (PPI) ${ }^{12}$, las publicaciones científicas en Venezuela se clasificarían en dos niveles: las tipo A, revistas que, en el caso de las ciencias sociales, pertenecen a índices como Clase y Latindex ${ }^{13}$; y las tipo B, publicaciones que no forman parte de los índices mencionados y que se ubican en y por encima del percentil 50, según un listado aprobado por el FONACIT, en su reunión 98 del 5 de mayo de $2005^{14}$.

Si se busca otro punto de referencia para un acercamiento a la calidad de los productos en ciencia política en Venezuela, y quizá por ello al prestigio de sus revistas, el indicador de las publicaciones de investigadores venezolanos en el Social Science Citation Index posiblemente arroje una perspectiva. El promedio anual de trabajos reseñados en este índice, sin contar con la información necesaria para discriminar por área, es de 42,78 (tabla 2), es decir, unos 3,5 artículos mensuales. 
TABLA 2: Publicaciones de investigadores venezolanos en el Social Science Citation Index

\begin{tabular}{lllllllllllllll}
\hline & 90 & 91 & 92 & 93 & 94 & 95 & 96 & 97 & 98 & 99 & 00 & 01 & 02 & 03 \\
\hline $\begin{array}{l}\text { Social } \\
\begin{array}{l}\text { Science } \\
\text { Citation } \\
\text { Index }\end{array}\end{array}$ & 28 & 35 & 25 & 39 & 25 & 39 & 80 & 56 & 48 & 43 & 38 & 72 & 39 & 32 \\
\hline
\end{tabular}

Fuente: http:// www.octi.gov.ve

Un último ángulo de visión de la calidad de las investigaciones en la disciplina puede hallarse en los premios a la productividad que otorga la Fundación para la Promoción del Investigador (FVPI), otra institución adscrita al Ministerio de Ciencia y Tecnología. El ya mencionado Programa de Promoción al Investigador (PPI) es una política pública que ofrece incentivos para la publicación en revistas indexadas (Tabla 3). Las convocatorias del PPI son anuales y, en general, consiste en un sistema de acreditación pública con evaluación de credenciales por pares. EI PPI clasifica a los investigadores en seis grupos: los candidatos, los niveles del uno al cuatro y los eméritos. Los criterios de clasificación son varios. El central fue referido: haber publicado en años recientes un acumulado de artículos en revistas tipo A y B. Otros lineamientos no menos importantes son: tener título de maestría y doctorado; ser responsable o estar adscrito a un programa de investigación científica financiado; contribuir a la formación de investigadores; demostrar evidencia de independencia en las tareas de investigación; tener el reconocimiento de experticia en el campo; y promover o liderar grupos de investigación en asuntos de alto impacto en el ámbito nacional o regional ${ }^{15}$.

TABLA 3: Premios por nivel de calificación (convocatoria 2005)

\begin{tabular}{crccc}
\hline & Bolívares & US\$ & Duración & Desembolso \\
\hline Candidato & 594.000 & 276 & 3 años & Trimestral \\
Nivel 1 & 1.189 .000 & 553 & 2 años & Trimestral \\
Nivel 2 & 1.784 .000 & 829 & 3 años & Trimestral \\
Nivel 3 & 2.484 .000 & 1.155 & 4 años & Trimestral \\
Nivel 4 & 3.184 .000 & 1.480 & 5 años & Trimestral \\
Emérito & 3.184 .000 & 1.480 & - & Trimestral \\
\hline
\end{tabular}

Para el 2004, de los 3189 investigadores adscritos al PPI y de los 889 que constituyen el grupo de ciencias sociales, 67 fueron catalogados como cientistas políticos. Éstos, a su vez, fueron reclasificados por líneas de investigación, con base en un clasificador temático desarrollado por la Unesco. Las cifras son entonces las siguientes: 7 investigadores en relaciones internacionales, 26 en ciencias políticas, 0 en ideologías políticas, 3 en instituciones políticas, 9 en vida política, 10 en sociología política, 0 en sistemas políticos y en teoría política, 5 en opinión pública, 2 en sistemas políticos en el área americana, 3 en otras especialidades y 2 no pudieron ser discriminados. Sobre estos politólogos puede decirse que: 34 son del sexo masculino y 33 del femenino; 63 son de Venezuela, 2 de Alemania, 1 es de los Estados Unidos y 1 es de Ecuador; 33 residen en el

15 Conultar: http:// www.ppi.org.ve/ programa/ convoca2005/ reglamento.htm 
estado Zulia, 14 en el Distrito Federal, 8 en Miranda, 7 en Mérida, 3 en Táchira, 1 en Anzoátegui y 1 en Carabobo.

Por último, un total de 14 de tales investigadores en ciencia política fueron ubicados por los comités de evaluación como candidatos (21\%), 17 en el nivel $1(25 \%), 20$ en el nivel $2(30 \%), 11$ en el nivel $3(16 \%)$ y 5 en el nivel $4(7 \%)^{16}$. Para obtener una visión adecuada del comportamiento de los cientistas políticos, cabe decir que las tendencias generales, tanto la población de investigadores adscritos al PPI como del grupo de cientistas sociales acreditados por el PPI para el 2004, indican que hay 983 y 370 candidatos (30,8 y 41,6\%), 1205 y 226 en el nivel 1 (37,7 y 25,4\%), 602 y 176 en el nivel 2 (18,8 y 19,7\%), 231 y 67 en el nivel 3 (7,2 y 7,5\%), 155 y 49 en el nivel 4 (4,8 y 5,5\%), así como 13 profesores y 1 profesor emérito $(0,4 \text { y } 0,1 \%)^{17}$. Los politólogos reflejan una orientación contraria a las tendencias de ambos grupos: se hallan con preferencia en los sectores centro y superior 0 , dicho de otro modo, se concentran menos en el segmento bajo de la clasificación.

Unas ideas finales que bien sirven de referencia, a pesar de que los datos no discriminan a los cientistas políticos. El PPI aplicó una encuesta entre octubre de 1996 y abril de 1997, entre la población de investigadores adscritos y no adscritos al programa ${ }^{18}$. En los datos recabados se halló que el PPI propicia el desarrollo de la carrera de investigador científico: sí (70\%), no (17\%), no opina (11\%). El PPI da más reconocimiento que la institución de origen: muy de acuerdo (36\%), algo de acuerdo (31\%), en desacuerdo (20\%), no opina (10\%), no responde (3\%). Uno de los efectos del PPI ha sido el aumento del número y calidad de las revistas científicas nacionales: muy de acuerdo (19\%), algo de acuerdo (28\%), en desacuerdo (32\%), no opina (19\%), no responde (2\%). El PPI fomenta la publicación en revistas internacionales de impacto: muy de acuerdo (52\%), algo de acuerdo $(28 \%)$, en desacuerdo $(7 \%)$, no opina $(9 \%)$, no responde (3\%). El PPI propicia la publicación en revistas de alto impacto para dar mayor visibilidad y legitimidad a la investigación nacional: muy de acuerdo (37\%), algo de acuerdo (32\%), en desacuerdo (17\%), no opina (10\%), no responde (3\%).

Otros datos de interés señalan que: Los criterios de ingreso y permanencia en el PPI reflejan la productividad y calidad de la investigación reciente, más que el historial del investigador: muy de acuerdo (44\%), algo de acuerdo (33\%), en desacuerdo (11\%), no opina (9\%), no responde (3\%). El PPI debe aplicar un escalafón similar al universitario por el que sólo se asciende: muy de acuerdo (16\%), algo de acuerdo (18\%), en desacuerdo (63\%), no opina (3\%), no responde (1\%) El hecho de tener un nombramiento de profesor o investigador en una institución nacional califica para ingresar al programa: muy de acuerdo (8\%), algo de acuerdo (13\%), en desacuerdo (75\%), no opina ( $2 \%)$, no responde $(2 \%)$.

Los entrevistados consideraron, además, que el monto de la beca es adecuado: muy de acuerdo (15\%), algo de acuerdo (28\%), en desacuerdo (47\%), no opina (8\%), no responde (2\%). El sistema universitario debería utilizar criterios similares a los del PPI para revertir la homologación remunerativa a la comunidad académica: sí $(71 \%)$, no (16\%), no opina (10\%), no responde (4\%). Las decisiones erróneas del PPI son más la excepción a la norma: muy de acuerdo (32\%), algo de acuerdo (27\%), en desacuerdo (8\%), sin opinión (32\%), no respondió (2\%). En relación con la revisión periódica de credenciales, se está: muy de acuerdo (73\%), algo de acuerdo (22\%), en desacuerdo (1\%), sin opinión (3\%), no respondió (1\%).

Consultar la Base de Datos de Investigadores Activos, en la Base de Datos/ Conexión On-Line, en http:// www.ppi.org.ve Tomado de: http:// www.ppi.org.ve/ programa/ resultados/ estadisticas/index.htm

Datos consultados en: http://www. ppi.org.ve/ investigadores/index.htm 


\section{La enseñanza de la disciplina en Venezuela}

Por otra parte, la tarea de describir con detalle el estado de la enseñanza de ciencia política en Venezuela se deja mirar a veces como una empresa infinita. Tantas universidades poseen varias facultades de ciencias sociales y humanidades que abren cada semestre distintas materias propias de la disciplina. La delimitación se hace inevitable y esta sección se centró en las ofertas de pregrado y postgrado que tenían al Estado como problema principal.

El estado de la disciplina revela un crecimiento en el país. La enseñanza dejó de ser una cuestión exclusiva de la capital. Ésta se encuentra en regiones como Aragua, Carabobo, Lara, Mérida, Táchira, Trujillo y Zulia. La cantidad mayor de ofertas de estudio se reporta en el postgrado y sobre ello cabe afirmar dos ideas. La preocupación central en este ámbito es el gobierno y la tendencia apunta hacia el profundizar la especialización: de las maestrías en gobierno y políticas públicas, se ha pasado a las especializaciones en gerencia pública y luego a los diplomas en gerencia de programas sociales o en auditoria del Estado. Por último, el mayor número de docentes y estudiantes está en el área de pregrado. Es decir, cada vez más cursantes de pregrados tienen más oportunidades de participar en postgrados en el país.

TABLA 4: Docentes y estudiantes venezolanos por centro de enseñanza

\begin{tabular}{|c|c|c|c|c|}
\hline & Universidad & Título & $\begin{array}{l}y \\
\text { है } \\
\tilde{D} \\
8\end{array}$ & 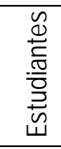 \\
\hline \multirow{9}{*}{$\begin{array}{l}\frac{0}{0} \\
\frac{0}{0} \\
\frac{0}{2}\end{array}$} & Universidad de Los Andes & 1. Licenciatura en Ciencias Políticas & 27 & 600 \\
\hline & $\begin{array}{l}\text { Universidad Bolivariana de } \\
\text { Venezuela }\end{array}$ & $\begin{array}{l}\text { 2. Licenciatura en Estudios Políticos } \\
\text { y de Gobierno }\end{array}$ & & \\
\hline & Universidad Central de Venezuela & $\begin{array}{l}\text { 3. Licenciatura en Ciencias Políticas y } \\
\text { Administrativas }\end{array}$ & 50 & 500 \\
\hline & & 4. Licenciatura en Estudios Internacionales & 80 & 900 \\
\hline & Universidad Fermín Toro & 5. Licenciatura en Ciencias Políticas & 55 & 63 \\
\hline & Universidad Rafael Urdaneta & 6. Licenciatura en Ciencias Políticas & 10 & 100 \\
\hline & Universidad Santa María & 7. Licenciatura en Estudios Internacionales & & \\
\hline & Universidad Valle del Momboy & 8. Licenciatura en Ciencias Políticas & 16 & 85 \\
\hline & Universidad del Zulia & 9. Licenciatura en Ciencias Políticas & & \\
\hline & $\begin{array}{l}\text { Instituto de Estudios Superiores y de } \\
\text { Administración }\end{array}$ & 1. Especialización en Gestión Pública & 15 & 27 \\
\hline \multirow{6}{*}{$\begin{array}{l}\frac{0}{0} \\
\frac{0}{0} \\
\text { ஸे } \\
0\end{array}$} & Instituto Zuliano de Estudios Políticos & $\begin{array}{l}\text { 2. Maestría en Dirección y Gestión Pública } \\
\text { Local para el Área Andina }\end{array}$ & & \\
\hline & & $\begin{array}{l}\text { 3. Programa en Gerencia de Procesos para } \\
\text { la Gestión Pública }\end{array}$ & & \\
\hline & & $\begin{array}{l}\text { 4. Programa de Planificación y Control de } \\
\text { Gestión para la Administración Pública }\end{array}$ & & \\
\hline & & 5. Programa de Formación de Gerentes Públicos & & \\
\hline & Universidad de los Andes & 6. Maestría en Ciencias Políticas & 12 & 60 \\
\hline & Universidad Bicentenaria de Aragua & $\begin{array}{l}\text { 7. Maestría en Ciencias Políticas, mención } \\
\text { Planificación del Desarrollo }\end{array}$ & 8 & 15 \\
\hline
\end{tabular}




\begin{tabular}{|c|c|c|c|}
\hline \multirow[t]{3}{*}{ Universidad de Carabobo } & 8. Diplomado en Gerencia Pública & 10 & 3 \\
\hline & 9. Especialización en Gerencia Pública & 12 & \\
\hline & $\begin{array}{l}\text { 10. Maestría en Ciencia Política y Administración } \\
\text { Pública }\end{array}$ & 12 & \\
\hline \multirow[t]{3}{*}{ Universidad Católica Andrés Bello } & $\begin{array}{l}\text { 11. Especialización en Gerencia de Programas } \\
\text { Sociales }\end{array}$ & 12 & \\
\hline & 12. Maestría en Gerencia de Programas Sociales & 12 & \\
\hline & $\begin{array}{l}13 \text { Diplomado en Gobernabilidad y Gerencia } \\
\text { Política }\end{array}$ & 55 & \\
\hline \multirow[t]{11}{*}{ Universidad Central de Venezuela } & $\begin{array}{l}\text { 14. Especialización en Sistemas y Procesos } \\
\text { Electorales }\end{array}$ & 12 & \\
\hline & 15. Especialización en Gobierno y Políticas Públicas & 12 & \\
\hline & 16. Doctorado en Ciencias Políticas & 10 & \\
\hline & 17. Doctorado en Estudios del Desarrollo & 6 & \\
\hline & $\begin{array}{l}\text { 18. Maestría en Planificación del Desarrollo, } \\
\text { mención Planificación Global }\end{array}$ & 17 & \\
\hline & $\begin{array}{l}\text { 19. Especialización en Planificación del Desarrollo, } \\
\text { mención Planificación Global }\end{array}$ & & \\
\hline & $\begin{array}{l}\text { 20. Maestría en Planificación del Desarrollo, } \\
\text { mención Política Social }\end{array}$ & 16 & \\
\hline & 21. Especialización en Seguridad Social & 11 & \\
\hline & 22. Maestría en Relaciones Internacionales & & \\
\hline & 23. Maestría en Seguridad Social & & \\
\hline & 24. Especialización en Seguridad Social & & \\
\hline $\begin{array}{l}\text { Universidad Centro Occidental } \\
\text { Lisandro Alvarado }\end{array}$ & $\begin{array}{l}\text { 25. Especialización en Gerencia de Programas } \\
\text { Sociales }\end{array}$ & 10 & \\
\hline \multirow[t]{3}{*}{ Universidad Metropolitana } & $\begin{array}{l}\text { 26. Diplomado en Gobernabilidad y Política } \\
\text { Exterior de Venezuela }\end{array}$ & 6 & \\
\hline & $\begin{array}{l}\text { 27. Diplomado en Gobernabilidad, Desarrollo y } \\
\text { Ciudadanía }\end{array}$ & 6 & \\
\hline & $\begin{array}{l}\text { 28. Diplomado en Gestión Municipal y } \\
\text { Gobernabilidad }\end{array}$ & 13 & \\
\hline \multirow{2}{*}{$\begin{array}{l}\text { Universidad Nacional Experimental } \\
\text { del Táchira }\end{array}$} & 29. Especialización en Gerencia Pública & 12 & \\
\hline & $\begin{array}{l}\text { 30. Diplomado en Control de la Administración } \\
\text { Pública }\end{array}$ & 6 & \\
\hline \multirow[t]{4}{*}{ Universidad Simón Bolívar } & $\begin{array}{l}\text { 31. Especialización en Opinión Pública y } \\
\text { Comunicación Política }\end{array}$ & 14 & \\
\hline & 32. Maestría en Ciencias Políticas & 27 & \\
\hline & 33. Doctorado en Ciencias Políticas & 27 & \\
\hline & 34. Especialización en Auditoría del Estado & 22 & \\
\hline Universidad Valle del Momboy & 35. Especialización en Gerencia Pública & 10 & \\
\hline \multirow[t]{2}{*}{ Universidad del Zulia } & 36. Maestría en Ciencias Políticas y Derecho Público & & \\
\hline & 37. Doctorado en Ciencias Políticas & & \\
\hline
\end{tabular}

Nota: Los datos expuestos en esta tabla corresponden al lapso septiembre 2004-febrero de 2005 y son un estimado de los docentes y estudiantes que, en general, asisten a las asignaturas. Los datos no distinguen la dedicación de los docentes, que puede ser exclusiva, tiempo completo, y medio tiempo. 


\section{Financiamiento de estudios de cuarto nivel}

Los politólogos venezolanos, en la actualidad, tienen un abanico de opciones para garantizar la viabilidad de los estudios de postgrado, tanto dentro como fuera del país. El Estado adelanta varias políticas con tales propósitos. La primera es la Fundación Gran Mariscal de Ayacucho (Fundayacucho $^{19}$, una institución adscrita al Ministerio de Educación Superior que ha diseñado varias políticas: el Programa de Financiamiento Educativo, que se compone del Programa de Crédito Educativo o Financiamiento Educativo y que es el proyecto central; los Programas ejecutados mediante convenios; el Programa de cooperación con universidades y demás instituciones de educación superior; los Programas de Cooperación Internacional; el Programa Ecos Nord, de cooperación entre centros de enseñanza e investigación venezolanos y franceses; y el Programa de fortalecimiento del Estado a través de la formación de talento humano para la gerencia pública.

El Fondo Nacional de Ciencia y Tecnología (FONACIT) ${ }^{20}$, organización perteneciente al Ministerio de Ciencia y Tecnología (MCT), ejecuta el Plan de Desarrollo de Talento Humano de Alto Nivel, que concede subvenciones para la realización de doctorados nacionales en áreas vinculadas con la calidad de la gestión pública. Para cursar maestrías y doctorados en el país, el mismo FONACIT implementa asimismo el Programa de Becas de Financiamiento para Estudios de Maestrías y Doctorado. Concebido como apoyo a la investigación, el proyecto se dirige especialmente a la formación, incorporación y actualización de investigadores.

Los consejos de desarrollo científico y humanístico de las distintas universidades venezolanas es, para los cientistas políticos, otra fuente de financiamiento de estudios de postgrado. EI CDCH de la Universidad Central de Venezuela ${ }^{21}$, por ejemplo, presenta la siguiente oferta de programas: una beca sueldo nacional o exterior, para profesores que deseen realizar maestrías, doctorados y post-doctorados; una beca académica para egresados, la cual cubre los postgrados a nivel nacional; una subvención matrícula, para profesores que se capaciten en la misma universidad; una beca sabática y una subvención de pasantía de estudio para profesores.

Fuera del sector gubernamental y universitario se encuentran un conjunto de opciones de apoyo financiero a la formación profesional de los politólogos. Las que más resaltan son las siguientes. El Programa de Becas Fulbright, de la Oficina Informativa y Cultural de la Embajada de los Estados Unidos, es una muestra de ello22. Este tipo de subvención representa una ayuda a profesores universitarios, en maestrías y doctorados. El Programa Sócrates-Erasmus y el Programa de becas de alto nivel de la Unión Europea para América Latina (Programa Alßan) son, asimismo, iniciativas que permiten los estudios de maestría y doctorado, así como de formación superior especializada 0 actualización profesional superior, en el marco de la enseñanza superior de la Unión Europea ${ }^{23}$. La Organización de Estados Americanos (OEA), mediante la Agencia Interamericana para la Cooperación y el Desarrollo (AICD), también ofrece un programa de subvención de estudios académicos y de actualización profesional, en los centros de formación del hemisferio ${ }^{24}$.

Ver: http://www.fgma.gov.ve, http://www.mes.gov.ve

Consultar: http://www.miproyecto.gov.ve, http://www.mct.gov.ve/, http://www.fonacit.gov.ve

Consultar: http://www.cdch-ucv.org.ve/

Consultar: http://embajadausa.org.ve

Ver: http:// www.programalban.org y http://desarrollo.universia.es

Ver: http://www.educoas.org/portal/es/becas/acerca.aspx?culture=es y http:// www.mpd.gov.ve/ 
Con el fin de cursar estudios de postgrado en el Reino Unido, la embajada británica presenta los programas de becas Chevening y Royal Holloway ${ }^{25}$. El primero es una iniciativa financiada por el Foreign and Commonwealth Office (FCO), con respaldo de universidades de ese país, y cubre los gastos de matrícula hasta por un año; el último, en cambio, forma parte de las políticas de subvención de la Royal Holloway, University of London. La Fundación Alexander von Humboldt desarrolla el programa Becas Humboldt y Georg Forster ${ }^{26}$, las cuales financian la realización de proyectos en algún centro de investigación en Alemania y se dirige por ello a los cientistas con grados académicos equivalentes a doctorados. De último se encuentra la Fundación Carolina y los Programas de "Becas MAE-AECl" del Ministerio de Asuntos Exteriores de España27. El uno sirve de apoyo a los estudios de postgrado e investigaciones en ese país, a través de cuatro programas: el de formación, el de investigación, el internacional de visitantes y el de responsabilidad social. El otro contempla becas para estudios de postgrado en centros universitarios públicos y privados de España.

\section{LOS POLITÓLOGOS EN LA SOCIEDAD VENEZOLANA DE HOY}

La politología como profesión enfrenta problemas de conocimiento y validez social en Venezuela: qué es y cuál es su utilidad son preguntas que aparecen en la sociedad. Al revisar la planilla de datos para el "Censo del Personal Activo, J ubilado y Pensionado de la Administración Pública" en noviembre de $2004^{28}$, se identifica a los estudios políticos y administrativos y los estudios internacionales como profesiones propias de las ciencias sociales. Ahora bien, cuando se chequea el formato de datos de los cargos reconocidos por el censo, el cual tiene 2166 entradas, sólo se encuentran las figuras de agregado en relaciones internacionales y analista de asuntos públicos.

Ello sorprende más si se considera que el principal empleador de los politólogos en Venezuela es el Estado. Las instituciones del gobierno nacional, la Asamblea Nacional y el Consejo Nacional Electoral han sido espacios tradicionales de trabajo para los politólogos. La descentralización de 1989 en Venezuela tuvo, en este sentido, repercusiones favorables en el campo laboral: ahora es usual hallar a los politólogos ejerciendo la profesión en alcaldías y gobernaciones.

Salvo encuestadoras y consultoras políticas, que no son muchas en el país, es difícil encontrar a un politólogo que ejerza la profesión en el sector privado. Las asociaciones civiles, las fundaciones 0 incluso las empresas privadas son territorios donde la profesión tiene escasa visibilidad y aceptación. Además, no es extraño acudir, por ejemplo, a una institución financiera y tener que seleccionar la opción otros al introducir el dato de la profesión.

Estas dificultades de reconocimiento de la profesión explican, en parte, la formulación y presentación ante la Asamblea Nacional de un anteproyecto de Ley de Ejercicio de la Politología, el cual se encuentra hoy en día en su segunda y última discusión ${ }^{29}$. El anteproyecto apunta en dos direcciones. Primero se define la profesión y determina a quiénes les compete su ejercicio, cuáles son las actividades que lleva a cabo y bajo qué condiciones debe practicarse la disciplina. Luego se diseña una institución gremial, con el nombre de Federación Venezolana de Colegios de Politólogos, y un Fondo de Previsión Social.

\footnotetext{
Ver: http://www.britishcouncil.org/es/venezuela

Ver: http://portal.cnti.ve/ estudiantes.html

Ver: http:// www.fundacioncarolina.es y http://becas.universia.edu.ve/

Ver: http://censompd.no-ip.org/

Ver: http:// www.asambleanacional.gov.ve/ns2/leyes.asp?id=508
} 
La viabilidad económica de la profesión es igual de precaria. Según el referido censo, el promedio de los sueldos de alto nivel en 2004 para funcionarios de la administración pública era de US\$ 435,97 , ello sin tomar en cuenta las bonificaciones propias de la contratación. El caso de las universidades no es demasiado diferente. Casi todos los centros de enseñanza de ciencia política en Venezuela son de carácter público o dependen en alguna medida del financiamiento estatal. Pues bien, de acuerdo con la tabla de sueldos y salarios vigente, un profesor a dedicación exclusiva en una universidad pública, con categoría instructor, asistente, agregado, asociado o titular, obtiene una mensualidad respectiva de US $\$ 440,540,665,831$ y 1040 (Tabla 5).

TABLA 5: Tablas de sueldos personal docente y de investigación de las universidades nacionales vigente desde el 01/01/03 (US\$)

\begin{tabular}{lcccc}
\hline $\begin{array}{c}\text { Categoría } \\
\text { Académica }\end{array}$ & $\begin{array}{c}\text { Dedicación } \\
\text { Exclusiva }\end{array}$ & $\begin{array}{c}\text { Tiempo } \\
\text { Completo }\end{array}$ & $\begin{array}{c}\text { Medio } \\
\text { Tiempo Hora/ }\end{array}$ & $\begin{array}{c}\text { Costo } \\
\text { Semana }\end{array}$ \\
\hline Instructor & 440 & 353 & 176 & 6 \\
Asistente & 540 & 417 & 208 & 7 \\
Agregado & 665 & 491 & 246 & 8 \\
Asociado & 831 & 585 & 292 & 10 \\
Titular & 1040 & 695 & 347 & 11 \\
\hline
\end{tabular}

Fuente: www.apucvipp.edu.ve

\section{CONCLUSIÓN}

La ciencia política venezolana reproduce las más notables líneas de división existentes en la disciplina en muchos otros países y, particularmente, de la Ciencia Política norteamericana. No obstante, al compartir una misma pregunta (los problemas de estabilidad del SPV) existen oportunidades para la integración 0, al menos, la complementación de los distintos enfoques. El más notable de estos esfuerzos se ha realizado mediante la creación de la Red de Estudios Políticos (REDPOL) que, con el apoyo financiero del Estado venezolano, ha podido realizar los tres más recientes estudios de cultura y comportamiento político, incluida la última ola de la Encuesta Mundial de Valores ${ }^{30}$.

REDPOL ha sido un paso importante en el proceso de institucionalización de la comunidad de cientistas políticos venezolanos. No obstante, su dependencia exclusiva del financiamiento público le ha hecho vulnerable tanto a las restricciones fiscales del gobierno como a los cambios de prioridades de los equipos rectores de la política de investigación a nivel nacional. Similarmente, la enorme dependencia de las universidades públicas respecto del fisco nacional también ha vulnerado el proceso de expansión y eventual consolidación de la ciencia política como una disciplina científica y, por tanto, crítica. Con la sola excepción del IESA, el resto de los más importantes centros de investigación y docencia dependen de universidades públicas que, por razones de su propio ineficiente modelo de financiamiento, como por sus conflictivas relaciones con las autoridades nacionales anteriores y actuales, han sufrido importantes reducciones presupuestarias en términos reales.

30 La Red de Estudios Políticos (REDPOL) está constituida por varias universidades venezolanas: IEP-UCV, CENDES-UCV, IESA, USB y LUZ. REDPOL ha funcionado gracias al patrocinio del hoy llamado Fondo Nacional de Investigaciones Científicas (FONACIT). Esta Red de Estudios Políticos ha coordinado investigaciones en materias como el comportamiento electoral y la cultura política del venezolano. En especial, ha trabajado tanto en la aplicación de encuestas de opinión como en la elaboración de bases de datos estadísticos. Un producto de este esfuerzo de integración es la obra siguiente: Carrasquero, J osé V., Maingón, Thaís y F. Welsch (2001). 
A esto se suma el hecho de que, tanto por su tradicional "parroquialismo" como por la importancia de la crisis y los cambios políticos que viene sufriendo el país desde 1993, los científicos políticos venezolanos no han podido prescindir de inmiscuirse en el análisis de las coyunturas y, en consecuencia, sus posiciones han influido y se han visto influidas por los agudos y polarizados debates políticos e ideológicos que dividen a las elites y a los ciudadanos. En este contexto de precariedad financiera, de aguda polarización y de severa amenaza a las libertades cívicas, el futuro de la disciplina resulta incierto.

Sin embargo, hay espacio para una nota final en clave de optimismo. Las consecuencias de las condiciones adversas que enfrenta la ciencia política actualmente en el país, podrían apuntar hacia un fortalecimiento de la conciencia sobre la importancia que tiene la política para la sociedad. Los resultados de la confrontación política, y la amplitud de sus efectos, quizás contribuyan a que los venezolanos miren en el futuro qué puede hacer la ciencia política para mejorar los diseños institucionales y, en consecuencia sus vidas como ciudadanos. La polarización política y las dificultades de sostener las libertades cívicas posiblemente está despertando el interés de cada vez más venezolanos por la ciencia política que puede dar respuesta a los agudos problemas institucionales del país. Por ello, no sería sorprendente un impulso de la disciplina en el mediano plazo, una vez superada la coyuntura actual.

\section{REFERENCIAS}

Álvarez, Angel E. (coord.). 1996. El Sistema Político Venezolano: Crisis y Transformaciones. Caracas: UCV.

Arístides. 1982. Partisanship and Floating Behavoir in Venezuela. PhD Dissertation, Nit.

Baloyra, Enrique y J ohn Martz. 1979. Political Attitudes in Venezuela. Societal Cleavages and Political Opinion. Austin: University of Texas Press.

Brewer Carías, Allan R. 1984. Fundamentos de la administración pública. Caracas: Editorial Jurídica Venezolana.

Bolívar, Adriana y Carlos Kohn (comp.). 1999. El discurso político venezolano: un estudio multidisciplinario. Caracas: Fondo Editorial Tropykos.

Caballero, Manuel. 1993. Gómez, el Tirano Liberal. Caracas: Monte Ávila.

Carrasquero, J osé V., Thaís Maingon y F. Welsch (eds.). 2001. Venezuela en transición: elecciones y democracia, 1998-2000. Caracas: Redpol.

Carrasquero, J ose Vicente. 1994. Legitimacy and Popular Support for the Democratic Political System in Venezuela (mimeo). University of Connecticut.

Carrera Damas, Germán. 1988. "Formulación definitiva del Proyecto Nacional: 1870-1900". Cuadernos Lagoven 3. Caracas: Serie Cuatro Repúblicas.

Castro Leiva, Luis. 1988. "El Dilema Octubrista: 1945-1987". Cuadernos Lagoven 4. Caracas: Serie Cuatro Repúblicas.

Comisión para la Reforma del Estado. 1992. Constitución y reforma. Un proyecto de Estado social y democrático de Derecho. Caracas: COPRE.

Comisión para la Reforma del Estado. 1989a. La Descentralización, una oportunidad para la democracia. Caracas: COPRE. Comisión para la Reforma del Estado. 1989b. El rol del Estado venezolano en una nueva estrategia económica. Caracas: COPRE. Ellner, Steve y D. Hellinger (eds.) 2003. Venezuelan Politics in the Chavez Era: Class, Polarization and Conflict. Boulder, Lynne Rienner.

Ellner, Steve. 1998.. Venezuela's Movimiento al Socialismo. From Guerrilla Defeat to Innovative Politics. Durham-North Carolina: Duke University Press.

García-Pelayo, Manuel. 1986. El Estado de partidos. Madrid: Alianza.

García-Pelayo, Manuel. 1982. Las transformaciones del Estado contemporáneo. Madrid: Alianza.

Kelly, J aneth. 2003.Políticas públicas en América Latina. Caracas: IESA.

Kelly, Janeth. 1995. "The Question of Inefficiency and Inequality: social Policy in Venezuela". En Lessons of the Venezuelan Experience, editado por L. Goodman L. et al. Washington, D.C.: The Woodrow Wilson Center Press/The J ohns Hopkins University Press, 283-310. 
Kornblith, Miriam. 1998. Venezuela en los noventa. Las crisis de la democracia. Caracas: IESA.

Levine, Daniel. 1973. Conflict and Political Change in Venezuela. New Jersey: Princeton University Press.

López Maya, Margarita, Luis Gómez Calcaño y Thaís Maigón. 1989. De Punto Fijo al Pacto Social. Desarrollo y Hegemonía en Venezuela. Caracas: Fondo Editorial Acta Científica Venezolana.

Márquez, Gustavo (comp.). 1993. Gasto público y distribución del ingreso en Venezuela. Caracas: IESA.

Molina, José E. y Carmen Pérez Baralt. 1999. "La democracia venezolana en una encrucijada: las elecciones nacionales y regionales de 1998". América Latina Hoy. Revista de Ciencias Sociales 21:29-40.

Molina, J osé E. y Carmen Pérez Baralt. 1998. "Evolution of the Party System in Venezuela 1946-1993". J ournal of Interamerican Studies and World Affairs 40, 2: 1-26.

Montero, Maritza. 1991. Ideología, alienación e identidad nacional. Caracas: UCV.

Naim, Moisés y Ramón Piñango. 1984. El Caso Venezuela. Una llusión de Armonía. Caracas: IESA.

Penniman, H. 1980. Veneazuela at the polls. The national election of 1978. Washington: American Enterprise Institute for Public Police Research.

Pino Iturrieta, Elías. 2003. Las ideas de los primeros venezolanos. Caracas: UCAB.

Polanco Alcántara, Tomás. 1990. Juan Vicente Gómez. Aproximación a una Biografía. Caracas: Grijalbo.

Ramos Jiménez, Alfredo. 1999. Comprender el Estado. Introducción a la Politología. Mérida: Centro de Investigaciones de Política Comparada.

Ramos J iménez, Alfredo. 1997. Las formas modernas de la política. Estudios sobre la democratización de América Latina. Mérida: Centro de Investigaciones de Política Comparada.

Rey, J uan Carlos. 1980. Problemas Socio-Políticos de América Latina. Caracas: Ateneo y Jurídica Venezolana.

Rey, Juan Carlos. 1972. "El Sistema de Partidos Venezolano". Politeia (I): 175-230.

Salamanca, Luis. 1995. Crisis de la Modernización y Crisis de la Democracia en Venezuela: Una Propuesta de Análisis. Caracas: ILDIS.

Silva Michelena, J osé A. (coord.). 1987. Venezuela hacia el 2000. Desafíos y Opciones. Caracas: Nuevas Sociedad.

Silva Michelena, José A. y Heinz R. Sonntag. 1979. El Proceso electoral de 1978. Su Perspectiva Histórica Estructural. Caracas: Ateneo de Caracas.

Silva Michelena, J osé A. 1967. Exploración en análisis y síntesis. Caracas: UCV.

Sonntag, Heinz R y Thaís Maingon. 1992. Venezuela: 4-F 1992. Un Análisis Sociopolítico. Caracas: Nueva Sociedad.

Soriano, Graciela. 1996. El personalismo político hispanoamericano del siglo XIX. Criterios y proposiciones metodológicas para su estudio. Caracas: Monte Ávila.

Sosa, Arturo. 1995. Rómulo Betancourt y el partido del pueblo (1937-1941). Caracas: Fundación Rómulo Betancourt.

Torres, Arístides. 1982. Partisanchip and floating behavior in Venezuela. MIT: Ph.D. dissertation.

Urbaneja, Diego Bautista. 1978. "Introducción Histórica al Sistema Político Venezolano". Politeia 7: 11-59.

Urbaneja, Diego Bautista. 1992. Pueblo y Petróleo en la Política Venezonala. Caracas: Cepet.

Velásquez, Ramón J. et al. 1979. Venezuela moderna: medio siglo de historia, 1926-1976. Caracas: Fundación Eugenio Mendoza-Ariel.

Vicente, J osé. 1994. Legitimacy and Popular Support for the Democratic Political System in Venezuela. University of Connecticut. Welsh, Federico. 1992. "Transformación de la Cultura Política". Nueva Sociedad 1212: 16-20.

Ángel Álvarez Díaz es profesor de ciencias políticas y administrativas de la Universidad Central de Venezuela (UCV). Ex director del Instituto de Estudios Políticos de la UCV, fue también Jefe de la Cátedra de Teoría Política de la Escuela de Estudios Políticos y Administrativos de la UCV. Coordinador del Postgrado en Procesos y Sistemas Electorales del Centro de Estudios de Postgrado de la Facultad de Ciencias J urídicas y Políticas de la UCV, ha sido Profesor Visitante en la Universidad de Salamanca, España, en varias oportunidades entre el año 2000 y el 2002. Actualmente goza de licencia y se encuentra realizando estudios en la Universidad de Notre Dame, en Estados Unidos.

(E-mail: aalvare3@.nd.edu)

Said Dahdah Antar es Licenciado en Ciencias Políticas y Administrativas de la Universidad Central de Venezuela (UCV), investigador del Instituto de Estudios Políticos de la UCV, profesor de la Escuela de Estudios Políticos de la UCV y profesor del Programa de Comunicación Social de la Dirección de Estudios de Postgrado, de la Universidad Católica Andrés Bello (UCAB). (E-mail: sda07@hotmail.com) 